\title{
Amamentação na primeira hora de vida, uma revisão de literatura
}

\author{
$\underline{\text { Romário Carneiro de oliveira1 }}^{1}$; Abdias Medeiros de Oliveira²; Suelly Pinto \\ ${\text { Teixeira de } \text { Morais }^{3} \text { e Vinicius Carneiro de Oliveira }}^{4}$ \\ 1. Bolsista CNPq, Graduando em Medicina, Universidade Estadual de Feira de Santana, \\ romariocarneiro.5@hotmail.com \\ 2. Orientador, Departamento de Saúde, Universidade Estadual de Feira de Santana, abdias@uefs.br \\ 3. Participante do Nupes, Departamento de Saúde, Universidade Federal do Reconcavo Baiano, \\ Suelly.pinto@terra.com.br \\ . 4. Bolsista CNPq, Graduando em Medicina, Universidade Estadual de Feira de Santana, \\ viniciuscarneiro.10@hotmail.com
}

PALAVRAS-CHAVE: amamentação, período pós parto e saúde materno infantil

\section{INTRODUÇÃOO}

O início da amamentação na primeira hora de vida está associado à maior duração do aleitamento materno ${ }^{1}$ e à redução das mortes infantis ${ }^{2}$. O colostro (leite dos primeiros dias) contém o fator epidérmico de crescimento, que acelera a maturação da mucosa intestinal, e fatores imunológicos bioativos que conferem proteção imunológica ao lactente, prevenindo infecções intestinais ${ }^{3}$.

O contato "pele a pele" entre mãe e bebê logo após o parto favorece a colonização da pele do recém-nascido pela microbiota da mãe, facilita a regulação da temperatura corporal, mantém os níveis de glicemia estáveis e contribui para a estabilidade cardiorrespiratória ${ }^{4}$. A sucção da mama logo após o nascimento estimula a secreção de prolactina que induz produção e ejeção do leite, e da ocitocina que promove a contração uterina que consequentemente previne sangramento puerperal ${ }^{5,6}$.

\section{MATERIAL E MÉTODOS OU METODOLOGIA (ou equivalente)}

A revisão de literatura foi realizada no ano de 2017 a 2018 a partir de pesquisas nas bases de dados online da Biblioteca Virtual de Saúde (Lilacs e Scielo) usando os termos de busca equivalentes a amamentação, saúde materno infantil e período pósparto.

$\mathrm{Na}$ identificação dos artigos adotou-se o seguinte critério de inclusão: pesquisas originais que avaliassem os fatores relacionados com o sucesso ou insucesso da amamentação na primeira hora de vida, ano de publicação a partir de 2005 e estudos publicados nos idiomas português, inglês e espanhol.

Foram encontrados 76 artigos relevantes, identificadas e selecionadas para análise, desses foram excluídos 56 artigos por não se adequarem aos critérios de inclusão. Ao final foram analisados 20 artigos, com os seguintes desenhos de estudo: observacional, descritivo, qualitativo, coorte e corte transversal. 
Uma melhor compreensão dos fatores que impedem o início oportuno da amamentação é importante para permitir uma implementação mais efetiva das políticas de promoção do aleitamento materno na primeira hora de vida. Dentre os estudos analisados a prevalência de AM na primeira hora de vida variou de 9,3\% a 81,2\%, tendo uma média de 55,1\%. A cidade com menor percentual foi Joao Pessoa (Paraiba) e a com maior Montes Claros (Minas gerais) ${ }^{8} 18$.

Vários fatores tiveram associação significativa com a falta da alimentação na primeira hora de vida, sendo eles: parto cesáreo, atraso no resultado do teste de HIV, alta rotatividade dos partos, recusa da paciente, cansaço por trabalho de parto prolongado, intercorrências maternas e fetais após o parto e recusa da mãe a amamentar $8,9,12,13,14,15,16,18,19,20,22,23,25,26$. Apenas um de todos os estudos não acharam relação entre o parto Cesáreo e a falta do AM na primeira hora de parto $^{12}$.

Alguns fatores foram protetores ao AM na primeira hora de vida, dentre eles o

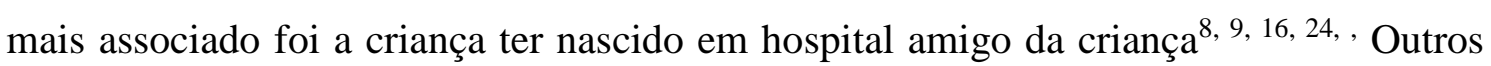
fatores protetores foram: multíparidade, cor não preta, parto normal, que fizeram prénatal, par, peso ao nascer igual ou superior a $2.500 \mathrm{~g}$ e que receberam ajuda da equipe de saúde para amamentar na sala de parto $^{13,19}$.

\section{CONSIDERAÇÕES FINAIS (ou Conclusão)}

O aleitamento materno é essencial para a saúde materno-infantil, amamentar na primeira hora de vida é muito importante diminuindo a mortalidade neonatal e hemorragia pósparto, além de aumentar o vinculo entre mãe e filho. Os resultados encontrados reforçam a necessidade de se continuar estimulando o aleitamento materno na primeira hora pós-parto, esta pratica está bastante inferior ao preconizado em muitos estudos. Para melhorar esta pratica é necessário protocolos e medidas publicas que tenham ações direcionadas aos fatores que diminuem a chance de uma criança seja amamentada na primeira hora de vida a exemplo do parto cesáreo.

\section{REFERÊNCIAS}

1- Moore ER, Anderson GC, Bergman N. Early skin-to-skin contact for mothers and their healthy newborn infants. Cochrane Database Syst Rev. 2012; (5).

2- Edmond KM, Zandoh C, Quigley MA, AmengaEtego S, Owusu-Agyei S, Kirkwood BR. Delayed breastfeeding initiation increases risk of neonatal mortality. Pediatrics. 2006; 117(3): 380-6.

3- Chirico GR, Marzollo S, Fonte CC, Gasparoni A. Anti-infective properties of human milk. J Nutr. 2008; 138(9):1801-6. 
4- Weiderpass E, Barros FC, Victora CG, Tomasi E, Halpern R. Incidência e duração da amamentação conforme o tipo de parto: estudo longitudinal no Sul do Brasil. Rev Saude Publica. 1998;32(3): 225-31.

5- Mercer JS, Erickson-Owens DA, Graves B, Haley MM. Evidence-based practices for the fetal to newborn transition. J Midwifery Womens Health. 2007; 52(3): 262-72.

6- Heinig MJ, Dewey KG. Health advantages of breast feeding for infants: a critical review. Nutr Res Rev. 1996;9(1):89-110.

7- Benatti AM, Demitto MO, Gramazio SL, Trindade RCA, Harumi HI, Ichisato SMT et al. Amamentação na primeira hora de vida: conhecimento e prática da equipe multiprofissional. av.enferm. 2017; 35( 1 ): 19-29.

8- $\quad$ Sampaio ARR, Bousquat A, Barros C. Skin-to-skin contact at birth: a challenge for promoting breastfeeding in a "Baby Friendly" public maternity hospital in Northeast Brazil. Epidemiol. Serv. Saúde. 2016, vol.25, n.2, pp.281-290.

9- Esteves TMB et al. Factors associated with late initiation of breastfeeding in the Brazilian Unified National Health System in the city of Rio de Janeiro, Brazil, 2009. Cad. Saúde Pública. 2015, vol.31, n.11, pp.2390-2400.

10- Boccplini, CS, Carvalho ML, Oliveira MIC, Perez ER. A amamentação na primeira hora de vida e mortalidade neonatal. J. Pediatr. (Rio J.). 2013, vol.89, n.2, pp.131-136.

11- Modesto SP, Devincenzi MU, Sigulem DM. Práticas alimentares e estado nutricional de crianças no segundo semestre de vida atendidas na rede pública de saúde. Rev. Nutr. 2018; 20 ( 4 ): 405-415.

12- Pillegi, Maria Cristina; Policastro, Adriana; Abramovici, Sulim; Cordioli, Eduardo; Deutsch, Alice D'Agostini. A amamentação na primeira hora de vida e a tecnologia moderna: prevalência e fatores limitantes / Breastfeeding in the first hour of life and modern technology: prevalence and limiting factors. Einstein (Säo Paulo). 2008, 6(4), pp. 467-472.

13- Pereira CRVR, Fonseca VM, Oliveira MIC, Souza IEO, Mello RR. Avaliação de fatores que interferem na amamentação na primeira hora de vida. Rev. bras. epidemiol. 2018. 16( 2 ), pp. 525-534.

14- Oliveira MIC, Silva KS, Gomes JSC, Fonseca VM. Resultado do teste rápido anti-HIV após o parto: uma ameaça à amamentação ao nascimento. Rev. Saúde Pública. 2010. 44( 1 ), pp. 60-69.

15- Boccolini CS, Carvalho ML, Oliveira MIC, Vasconcellos AGG. Fatores associados à amamentação na primeira hora de vida. Rev. Saúde Pública. 2011; 45( 1 ), pp. 69-78.

16- Silva MB, Albernaz EP, Mascarenhas MLW, Silveira RB. Influência do apoio à amamentação sobre o aleitamento materno exclusivo dos bebês no primeiro mês de vida e nascidos na cidade de Pelotas, Rio Grande do Sul, Brasil. Rev. Bras. Saude Mater. Infant. 2008; 8( 3 ), pp. 275-284.

17- Venancio SI, Escuder MML, Saldiva SRDM, Giugliani ERJ. A prática do aleitamento materno nas capitais brasileiras e Distrito Federal: situação atual e avanços. J. Pediatr. (Rio J.). 2010; 86( 4 ),pp. 317-324. 
18- Souza SCO, Paiva PA, Costa SM, Lacerda MKS, Pereira, Matheus Mendes; Gonçalves, Jaqueline TT. Aleitamento materno de crianças cadastradas na atenção primária à saúde. 2017; Rev. enferm. UFPE;11(supl.9), PP. 3583-3589.

19- Silveira RB, AE, Zuccheto LM. Fatores associados ao início da amamentação em uma cidade do sul do Brasil. Rev. Bras. Saude Mater. Infant. 2008; 8( 1 ), PP. 3543.

20- Bandeira SNN, Gubert MB, Santos W, Santos LMP. Fatores ligados aos serviços de saúde determinam o aleitamento materno na primeira hora de vida no Distrito Federal, Brasil, 2011. Rev. bras. epidemiol. 2016; 19 ( 3 ), pp. 509-524.

21- Vieira GO, Reis MR, Vieira TO, Oliveira NF, Silva LR, Giugliani ERJ. Tendência dos indicadores de aleitamento materno em uma cidade do Nordeste brasileiro. J. Pediatr. (Rio J.) 2015; 91( 3 ), pp. 270-277.

22- Monteiro, Juliana Cristina dos Santos; Nakano, Ana Márcia Spanó; Gomes, Flávia Azevedo. Amamentação precoce na primeira meia hora de vida da criança. Rev. Enferm. UERJ. 2006; 14(2), pp. 202-207.

23- Teles JM, Bonilha ALL, Gonçalves AC, Santo LCE, Marioto MDM. Amamentação no período de transição neonatal em Hospital Amigo da Criança. Rev. Eletr. Enf. 2015; 17(1), pp. 94-9

24- Moreira MEL, Gama SGN, Pereira APE, Silva AAM, Lansky S, Pinheiro RS et al. Clinical practices in the hospital care of healthy newborn infant in Brazil. Cad. Saúde Pública. 2014; 30( Suppl 1 ), pp. 128-139.

25- Belo MNM, Azevedo TÁCC, Belo MPM, Serva VMBDBF, Malaquias Figueiroa JN, Caminha MFC et al . Aleitamento materno na primeira hora de vida em um Hospital Amigo da Criança: prevalência, fatores associados e razões para sua não ocorrência. Rev. Bras. Saude Mater. Infant. 2014; 14( 1 ), pp 65-72.

26- Will TK, Arndt JG, Torres GG, Andrade JR, Pereira TSS, Molina MDCB. Fatores de proteção para a amamentação na primeira hora de vida. Rev Bras Promoc Saude. 2013; 26(2), pp. 261-267. 